\title{
Study on Mathematical Modeling and Experiment of Inductive Magnetic-gas Mixed Suspension Spherical Joint
}

\author{
Li Zeng ${ }^{\text {a, }}$, Zhida Zhu ${ }^{\mathrm{a}}$, Jin Sun ${ }^{\mathrm{a}}$, Zhang Fan ${ }^{\mathrm{a}}$, Lifeng Zhang ${ }^{\mathrm{b}}$ \\ ${ }^{a}$ College of Mechanical Engineering, Yangzhou University, Yangzhou 225127, China \\ ${ }^{\mathrm{b}}$ Department of Electrical Engineering and Electronics, Kyushu Institute of Technology, Fukuoka, Japan
}

\begin{abstract}
The multi-degree-of-freedom inductive spherical driving joint owns high mechanical integrity and has advantages such as control and trajectory planning. Because of its limit of rotor pose, instant stepping angle and inability to spin around any axis through center of sphere, the suspension and rotation precision of spherical reluctance driving joint isn't high and the response speed can't reach the best. Based on mechanism of rotation and suspension, due to distribution of air gap magnetic induction intensity, a novel inductive maglev spherical driving joint was presented, and electromagnetic suspending force and electromagnetic torque were established. Analyze the magnetic induction intensity, electromagnetic suspending force and electromagnetic torque through finite element simulation, build a bench test of maglev spherical driving joint, and do testing research on displacement characters and suspension and rotation characters of joint rotor.
\end{abstract}

Keywords: Magnetic-gas mixed suspension, Spherical joint, Displacement character, Suspending force character, Electromagnetic torque character.

\section{Introduction}

Robots and mechanical arms are usually driven by multiple single-degree of freedom devices and complex transmission mechanism. They gain three-dimensional motion, which lead to a serious of problems ${ }^{(1,2)}$, such as the increase of complexity, big volume, serious wear of friction surface of each joint, low efficiency, slow response and poor dynamic performance. As an entry point of research, the study of the system of magnetic levitation spherical driving joint can simplify the mechanism, realize the aim of no friction between rotor and stator and no wear of suspending supporting, improve the positional accuracy of joint and response speed and reduce the volume of mechanism to obtain high response speed. In this research field, magnetic levitation spherical motor and driving joint were first introduced in the world and a number of national patents were applied ${ }^{(3,4)}$. The research work has a study of mechanism of spherical surface reluctance motor's maglev spherical reluctance driving joint ${ }^{(5)}$, builds magnetic field flux and electromagnetic suspending force and electromagnetic torque model of joint ${ }^{(6,7)}$, and establishes joint ideal spherical rotor three-dimensional dynamics nonlinear coupling model and linearization of decoupling control is performed ${ }^{(8,9)}$. With the limit of rotor pose, instant stepping angle and inability to spin around any axis through center of sphere, its suspension and rotation precision of spherical reluctance driving joint is insufficient. In order to make response speed does not reach the fastest, the final pose of end effectors of joint have to be determined according to the planned track in advance. Therefore, a novel inductive maglev spherical driving joint was presented. Study mechanism of rotation and suspension and establish a mathematical model of joint system. Analyze the suspension and rotation characters through finite element simulation and testing.

\section{Maglev Spherical Joint Basic Composition}

The 3-D structural drawing of stator and rotor of inductive magnetic levitation spherical driving joint is shown in Fig.1. Stator 1 and 3 are spherically symmetric and keep coaxial, and they are with functions of driving rotor spin around stator axle and generating maglev force which is able to make rotor along the direction of stator axle. Stator 2 and 4 are spherically symmetric and keep coaxial, and their roles are driving rotor spin around its axle and producing maglev force which can make rotor stable suspending along the direction of rotor axle. Stator 5 was arranged on the 
bottom of spherical rotor, and its effects are driving spherical rotor spin around stator axle and producing maglev force which can make rotor stably suspend along the direction of stator axle. Every stator axle is mutually orthogonal, which produce an intersection that is center of sphere composed by every stator salient pole spherical enveloping surface.

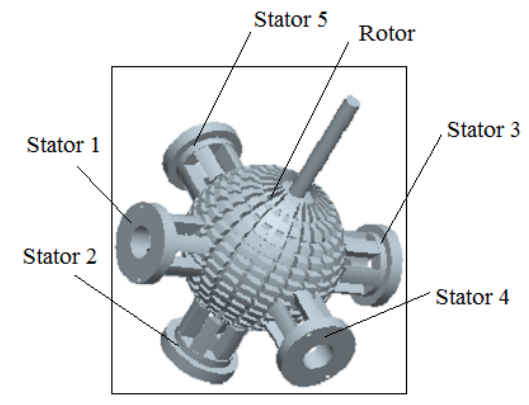

Fig.1. Schematic diagram of maglev spherical driving joint

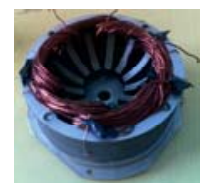

(a) Stator

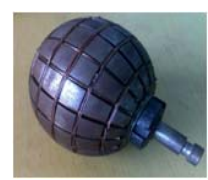

(b) Rotor

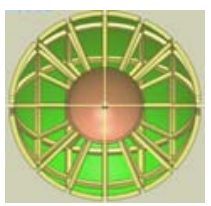

(c) Rotor's armature structure
Fig.2. Stator/Rotor structure

The structural drawing of inductive magnetic levitation spherical driving joint's rotor and stator is shown in Fig. 2. The stator is stacked by $50 \mathrm{~W} 470$ silicon steel sheet. There is a groove, with concentrated distribution winding method, in the middle of stator, embedded with three-phase winding whose connection mode is Y shape. When there is electricity, each phase winding not only generated electromagnetic torque that can drive rotor revolve but also support radial maglev force for rotor. Spherical rotor, compacted on axle by round nut and shaft shoulder, is stacked also by $50 \mathrm{~W} 470$ silicon steel sheet. On the surface of rotor, there is a mutually orthogonal groove. In the orthogonal point along radial direction to the centre of sphere, there is a hole. The $\mathrm{Cu}$ or $\mathrm{Al}$ is injected into groove and hole and then the armature structure of emanate orthogonal spherical cage shape will be formed in the core of centre of sphere short sub and intersect.

\section{Maglev Spherical Joint Mathematical Model}

\subsection{Joint suspending force and electromagnetic torque model}

(1) Joint air gap magnetic induction intensity's regularities of distribution
The magnetic potential is generated by each phase current of three-phase stator winding of maglev spherical driving joint in the way of sine or cosine distribution. By ignoring ultra harmonics component, groove leakage inductance, end region leakage inductance and magnetic saturation. A three-phase fundamental wave combining magnetic induction intensity ${ }^{(10)}$ came over three-phase winding magnetic potential of a stator, whose air gap of magnetic field between stator and rotor is figured out. That is defined as

$$
B_{s}(\theta, t)=B_{m} \cos (\omega t-p \cdot \theta)
$$

Where $\theta$ is the (tangential) angle of rolling stator axle, $\mathrm{Bm}$ is magnetic induction intensity amplitude, which depend on winding incoming current and air gap length and $p$ is magnetic field number of pole pairs generated by joint winding.

The magnetic flow of inductive magnetic levitation driving joint stator winding cut rotor spherical cage armature winding in space and induct ac electromotive force and electricity in the rotor. The rotating flux is produced in air gap as ac electricity pass rotor. Its magnetic induction intensity is defined as

$$
B_{r}(\theta, t)=K \cdot B_{m} \cos (\omega t-p \cdot \theta-\alpha)
$$

Where $K$ is inductance coefficient of joint stator and rotor, $\alpha$ is phase (including rotor rotating angular displacement) difference between rotor winding and stator winding rotating magnetic field. $K$ and $\alpha$ all depend on structure and slip ratio of inductive magnetic levitation driving joint stator, rotor. The total magnetic induction intensity generated by stator winding and rotor winding in peripheral air gap is defined as

$$
\begin{aligned}
B(\theta, t) & =B_{s}(\theta, t)+B_{r}(\theta, t) \\
& =B_{m}(\cos (\omega t-p \theta)+K \cos (\omega t-p \theta-\alpha))
\end{aligned}
$$

As shown in Eq.(3), distribution regularities of magnetic induction intensity of inductive magnetic levitation spherical driving joint air gap are relating to magnetic field number of pole pairs generated by stator winding, tangential angular position rotating stator axle and phase difference between rotor and stator rotating magnetic field.

(2) Distribution regularities of joint air gap magnetic energy

The envelope radius of the sphere of stator salient pole is $R$, the envelope radius of the sphere of rotor salient pole is $R_{r}$, the air gap between inner diameter of stator salient pole and external diameter of rotor salient pole is $\mathrm{g}_{0}=R-R_{r}$, if 
rotor centre of sphere of magnetic levitation spherical joint has no deviation. A rectangular coordinate system $\mathrm{f}(\mathrm{X}, \mathrm{Y}$, Z) and coordinate system $F(R, \varphi, \theta)$ (see Fig.3) are built by adopting a stator axle coincides $z$ axle. If rotating virtual displacement generated by rotor rotates around stator axle is $\Delta \alpha$, radial virtual displacement is $e=i x+j y+k z$, that is to say rotor centre of sphere moves from $\mathrm{O}(0,0,0)$ point to $\mathrm{O}_{1}(\mathrm{x}, \mathrm{y}$, $\mathrm{x})$ point, as $R_{r}>>\mathrm{x}, R_{r}>>\mathrm{y}, R_{r}>>\mathrm{z}$, the change rules of radial air gap length between stator and rotor salient pole envelope surface on optional position of stator magnetic pole envelope annularly spherical surface can be expressed by :

$$
g_{c}(\varphi)=g_{0}-x \cos \theta \sin \varphi-y \sin \theta \sin \varphi-z \cos \varphi
$$

The plane between stator and rotor salient pole is regarded as parallel plane, because the position deviated from equilibrium position is very small. The reluctance of working air gap is only considered in the whole magnetic circuit system by ignoring iron core, reluctance and eddy-current loss. The enveloping spherical crown on the stator pole, when air gap is $g_{c}(\varphi)$, take a tiny sphere, surround stator axis, which width is $R d \varphi$, length is $R \cos \varphi d \theta$ area is $d A=(R \cos \varphi d \theta)(R d \varphi), \quad\left(\varphi_{1}<\varphi<\varphi_{2}, 0<\theta<2 \pi\right)$, air gap tiny unit volume is $d V=g c(\varphi)(R \cos \varphi d \theta)(R d \varphi)$.
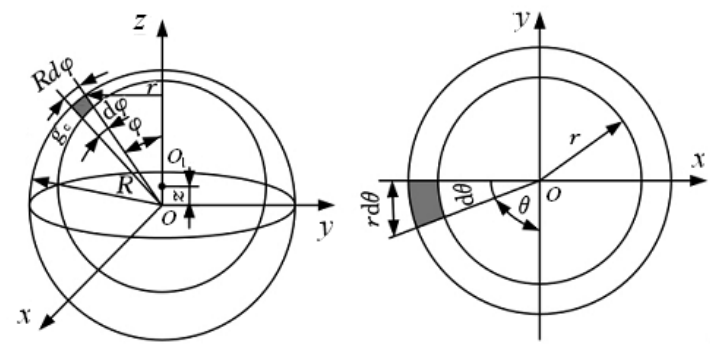

Fig.3. Schematic diagram of change of rotor air gap

According to the theory, magnetic field permeability of vacuum is $\mu 0$ then the total stored energy of joint air gap magnetic field is defined as

$$
\begin{aligned}
& W=\int_{0}^{V} \frac{B^{2}(\theta, t)}{2 \mu_{0}} d V \\
& =\frac{R^{2}}{2 \mu_{0}} \int_{\varphi_{2}}^{\varphi_{1}}\left(g_{0}-x \cos \theta \sin \varphi-y \sin \theta \sin \varphi\right. \\
& -z \cos \varphi) \cos \varphi d \varphi \int_{0}^{2 \pi}\left(B_{m}(\cos (\omega t-p \cdot \theta)\right. \\
& +K \cos (\omega t-p \cdot \theta-\alpha-\Delta \alpha)))^{2} d \theta
\end{aligned}
$$

If joint stator parameter is $\varphi_{1}=0, \varphi_{2}=\pi / 4$, then air gap total magnetic energy is defined as:

$$
W=\frac{\pi R^{2} B_{m}^{2}}{2 \mu_{0}}\left(1+2 K \cos (\alpha+\Delta \alpha)+K^{2}\right)\left(\frac{2+\pi}{8} z-\frac{\sqrt{2} g_{0}}{2}\right)
$$

(3)The distribution regularities of joint electromagnetic suspending force and electromagnetic torque
According to principle of virtual displacement and Eq.(6), radial virtual displacement $z$ and rotating virtual displacement $\Delta \alpha$ are in derivative respectively. Consequently, electromagnetic suspending force along direction of stator axle and electromagnetic torque rotated around stator axle, generated by a stator of magnetic levitation spherical driving joint, are got. That is defined as

$$
\left\{\begin{array}{l}
F=\left.\frac{\partial W}{\partial z}\right|_{\substack{z=0 \\
\Delta \alpha=0}}=\frac{\pi R^{2} B_{m}^{2}(2+\pi)}{16 \mu_{0}}\left(1+2 K \cos \alpha+K^{2}\right) \\
T=\left.\frac{\partial W}{\partial(\Delta \alpha)}\right|_{\substack{z=0 \\
\Delta \alpha=0}}=\frac{\sqrt{2} g_{0} K \pi R^{2} B_{m}^{2}}{2 \mu_{0}} \sin \alpha
\end{array}\right.
$$

As Eq.(7) is illustrated, magnetic induction intensity is defined as

$$
B_{m}=\frac{\mu_{0} N I}{g}
$$

Where $N$ is joint stator number of turns of winding, $I$ is current amplitude in flowed into stator winding and $\mathrm{g}$ is air gap length between spherical rotor and certain joint stator. As Eq. (7) is shown, the distribution regularities of electromagnetic suspending force and electromagnetic torque generated by inductive magnetic levitation spherical driving joint is cosine and sine along direction of rotating. The amplitude of electromagnetic suspending force and electromagnetic torque has the direct ratio to square of magnetic induction intensity $\mathrm{Bm}$ (have the inverse ratio to square of air gap length $g$ ).

(4) Joint rotor dynamics equation

According to Newton's second law, a kinetic equation of suspension and rotating of spherical rotor driven by a stator can be defined as

$$
\left\{\begin{array}{l}
M \cdot \frac{d^{2} z}{d t^{2}}=\frac{\pi R^{2}(2+\pi)\left(1+2 K \cos \alpha+K^{2}\right)}{16 \mu_{0}} B_{m}^{2}+F_{d} \\
J \cdot \frac{d^{2} \theta}{d t^{2}}=\frac{\sqrt{2} g_{0} K \pi R^{2} \sin \alpha}{2 \mu_{0}} B_{m}^{2}+M_{d}
\end{array}\right.
$$

Where $M$ and $J$ respectively are spherical rotor mass and rotational inertia, $F_{d}$ and $M_{d}$ separately are disturbing force and moment besides spherical rotors are under electromagnetic suspending force/ torque.

If spherical rotor deviation is $z$, under spherical rotors are interfered. In order to recover spherical rotor in balance state and keep air gap length is $\mathrm{g}_{0}$, controller should be adjusted to current of stator winding is magnified or decreased $I=I_{0} \pm \Delta I$. Then, magnetic induction intensity of air gap in equation mentioned is defined as

$$
B_{m}=\frac{\mu_{0} N\left(I_{0} \pm \Delta I\right)}{g_{0} \pm z}
$$


When rotors are interfered and their location are changed, winding current changes with that but magnetic induction intensity remain unchanged. Therefore, stability of joint electromagnetic torque nearly doesn't be influenced.

\section{Simulation Experimentation of Magnetic Levitation Spherical Joint}

\subsection{Air gap magnetic field character of magnetic levitation spherical driving joint}

(1) Finite element analysis model

Structure parameters of magnetic levitation spherical driving joint are given in Table 1.

Table 1. Structure parameters of magnetic levitation spherical driving joint

\begin{tabular}{|c|c|}
\hline Stator outer diameter $60 \mathrm{~mm}$ & $\begin{array}{c}\text { Centre of sphere to stator undersurface } \\
47 \mathrm{~mm}\end{array}$ \\
\hline $\begin{array}{c}\text { Stator small inner diameter } \\
7.5 \mathrm{~mm}\end{array}$ & Rotor longitude number of slots 18 \\
\hline Stator number of slots 12 & Rotor longitude number of slots 18 \\
\hline Stator slot radius $52 \mathrm{~mm}$ & $\begin{array}{c}\text { Rotor latitude number of slots radius } \\
5 \mathrm{~mm}\end{array}$ \\
\hline Stator groove radius $38.5 \mathrm{~mm}$ & Rotor sphere endocoele $10 \mathrm{~mm}$ \\
\hline Stator thickness $37 \mathrm{~mm}$ & Air gap of stator and rotor $1 \mathrm{~mm}$ \\
\hline
\end{tabular}

Geometric mesh model of stator and rotor of magnetic levitation spherical driving joint can be built (see Fig.4) by Table 1 parameters. It border subdivision picture is magnified, as Fig.5 shows.

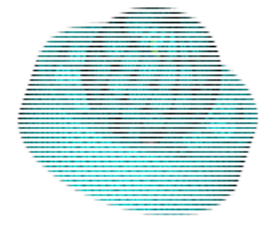

Fig.4. Mesh generation

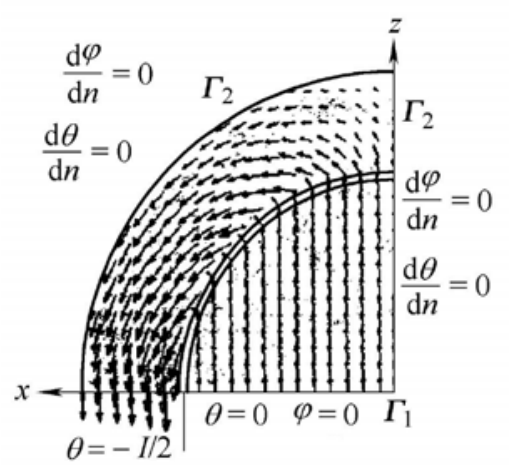

Fig.6. Boundary conditions of joint model

In finite element model analysis of magnetic levitation spherical driving joint, magnetic line of force which boundary conditions are shown as Fig.6 can be considered completely within model, since stator and rotor cores are stacked by silicon steel sheet. The differential quotient of flux linkage and magnetic flow of on spherical rotor core intermediate shaft and stator outer surface all are zero.

(2) Distribution regularities of joint air gap magnetic line of force and magnetic induction

The simulated chart of magnetic levitation spherical driving joint can be got, through

solving finite element model of magnetic levitation spherical driving joint. Fig.7 is magnetic line of force distribution of magnetic field. Fig. 8 is $2 \mathrm{D}$ inductive cloud picture of half section cut model of magnetic levitation spherical driving joint.

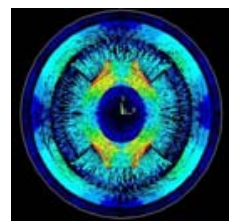

Fig.7 Magnetic line

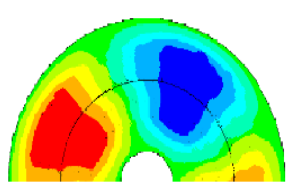

Fig.8 2D inductive cloud picture

From the color of Fig.8, the magnetic induction intensity of air gap of maglev spherical driving joint basically has a distribution of sine or cosine along the circle of the air gap, as shown in Figs. 9 and 10 shows the actual distribution curve of magnetic induction intensity. The curve of average distribution and the theoretical analysis are consistent. The magnetic induction intensity around the circle of air gap has two positive value points and two negative peak points, which coincide with the quadruple magnetic field.

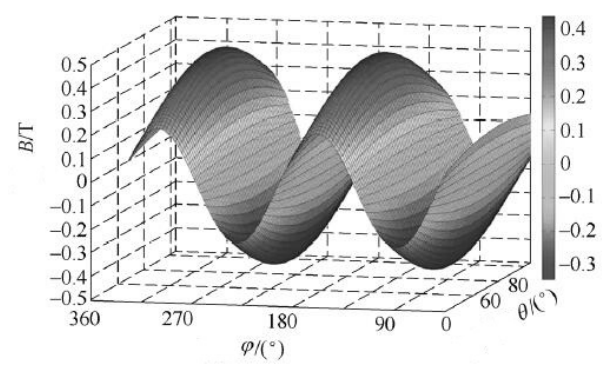

Fig.9. Distribution of magnetic flux density of air gap

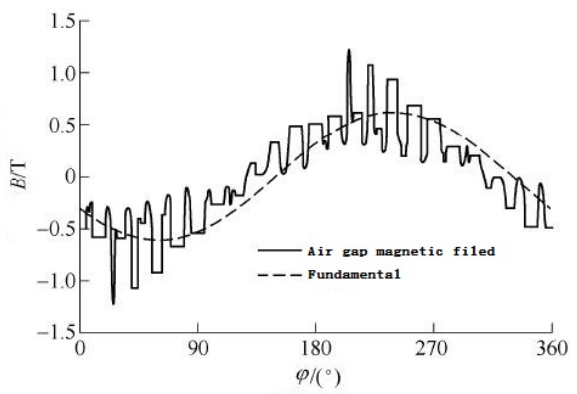

Fig.10. Relation of magnetic flux density and angle 


\subsection{The characteristics of the electromagnetic levitation force and torque of maglev spherical driving joint}

(1) The distribution of the electromagnetic levitation force and torque along with the corner

The key point of the finite element analysis to verify the mechanism of maglev spherical driving joint is to analyze whether maglev spherical driving joint can produce electromagnetic levitation force and torque. Fig.11 shows the distribution law of electromagnetic levitation force, produced at the air gap of maglev spherical driving joint, in the rotating direction of rotor. Fig.12 shows the distribution law of electromagnetic torque, produced by maglev spherical driving joint, in the rotating direction of rotor.

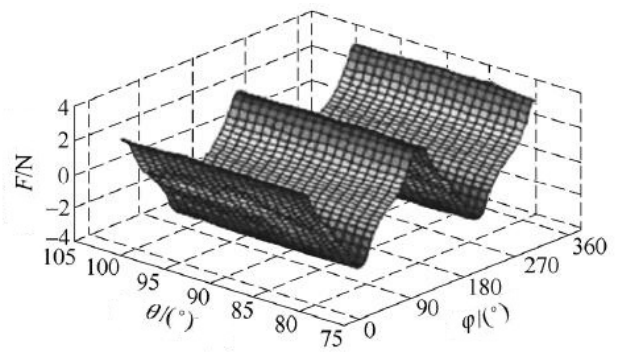

Fig.11 Distribution law of electromagnetic levitation force

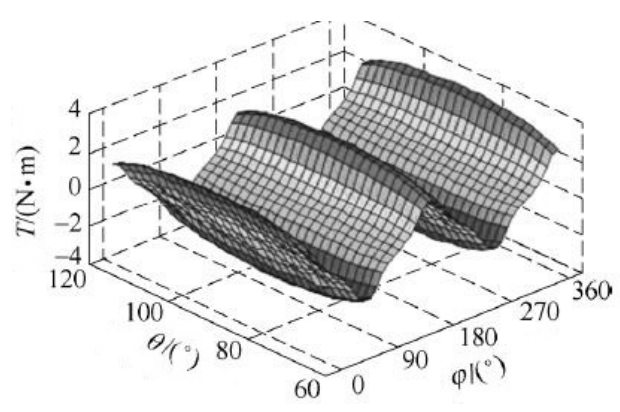

Fig.12 Distribution law of electromagnetic torque

(2) Distribution of electromagnetic levitation force and torque along with the air gap length

Fig.13 shows the relation curve of the force and torque and the air gap, when the input-current of stator winding is $2 \mathrm{~A}$ and the joint rotor spins around the central axis of sphere. Because of the fringe effect of stator teeth and grooves and flux leakage, the values of suspending force or electromagnetic torque in the same air gap are different. However, with the increase of the length of air gap, the suspending force and electromagnetic torque of the joint stator parameter both decrease.

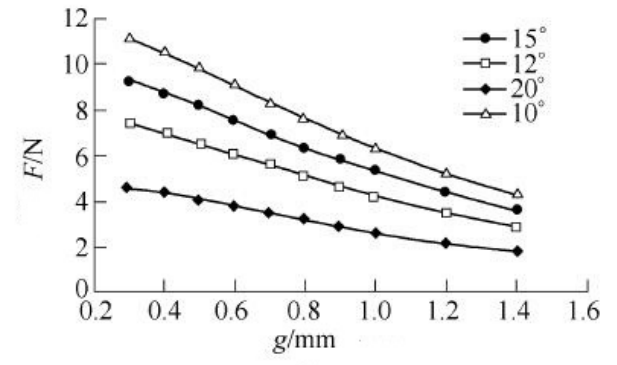

(a) electromagnetic levitation force

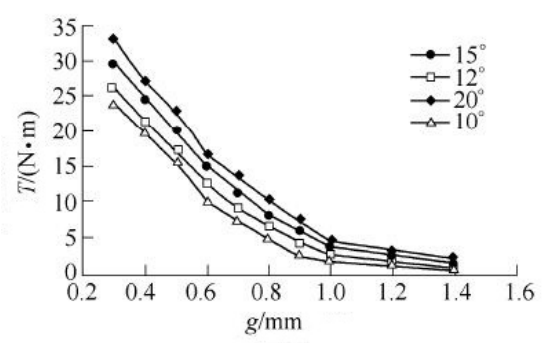

(b) electromagnetic torque

Fig.13. Relation of electromagnetic levitation force / electromagnetic torque and air gap $(I=2 \mathrm{~A})$

\section{Experimental Study of Maglev Spherical Driving Joint}

\subsection{Experimental facilities of maglev spherical driving joint}

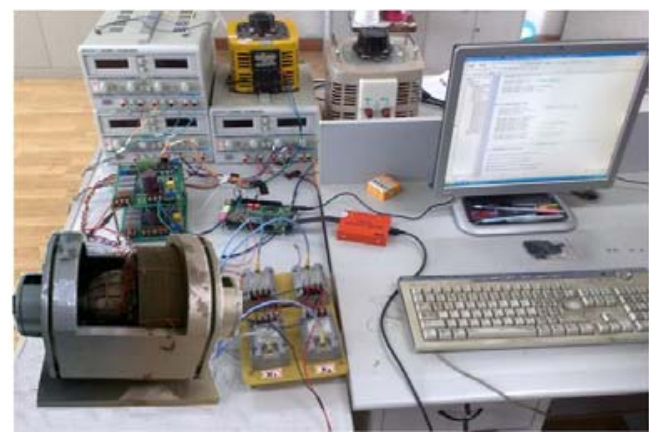

Fig.14. Joint prototype control system

Design a control-driven system of joint based on the verification of the characteristics of rotation and suspension of inductive maglev spherical driving joint. The system makes a differential drive of spherical rotor through two stators up and down, which makes the rotor to suspend and rotate. The control of rotor's rotating and suspending around $\mathrm{z}$-axes is based on the closed-loop control of motor control system: Input the command signal of angular displacement $\theta^{*}$ and the feedback signal of the rotor's angular displacement $\theta r$. Compare the two signals and get the deviation signal, which will be converted to rotational velocity signal $\omega^{*}$ of rotor through angular displacement controller. Comparing $\omega^{*}$ and the feedback signal of the rotor's actual angular velocity, deviation signal of rotational 
velocity can be got. It will be converted to rotate speed (or torque) to control current $i_{q}{ }^{*}$ by angular velocity controller. At the same time, input the control current signal $i_{d}{ }^{*}$ of suspending force. The signal will be converted to two-phase control voltage by current controller and then be transformed to switch power (inverter) control voltage by a static/ active $((d, q) /(\alpha, \beta))$ transformation of coordinates and 2-phase/ 3-phase $(2 \Phi / 3 \Phi)$ transformation. The voltage will control the input-current of joint windings and adjust the radial displacement (electromagnetic levitation force) and angular displacement (electromagnetic torque) of rotor. Command signal $\theta^{*}$ is used to control rotating angular displacement of rotor while command signal $z^{*}$ is used to control radical displacement.

Based on the above control system, establish the control experimental facilities of inductive maglev spherical driving joint as Shown in Fig.14.

\subsection{Experiments to get the characteristics of maglev spherical driving joint}

(1) Displacement characteristics of spherical rotor

Fig.15 shows the oscillogram of rotor when it is floating. There is on load on the driving joint. The designed air gap between rotor and stator is $0.6 \mathrm{~mm}$. The primary clearance of rotor's offset downward is $0.1 \mathrm{~mm}$. With the action of suspending control, spherical rotor will move to the center of stator, and achieve stable suspension. Rotor's maximum fluctuating peak value is $8 \mu \mathrm{m}$ when stably suspending.

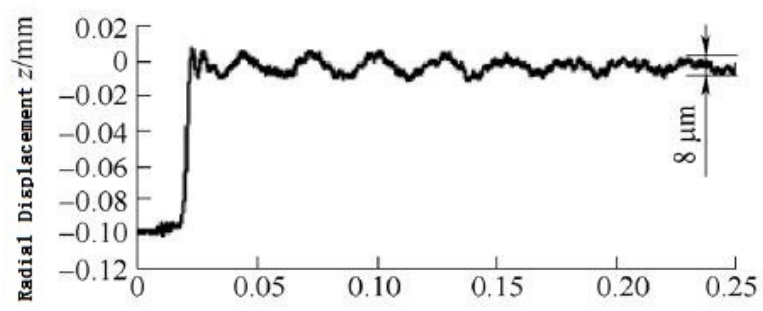

Fig.15. Floating property of rotor's radical displacemen

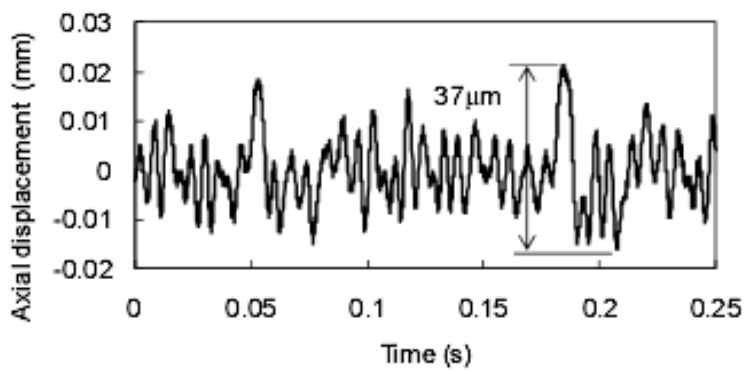

Fig.16. Vibration property of rotor's displacement

Fig.16 shows the wave diagram of displacement of rotor when the rotating speed is $1400 \mathrm{r} / \mathrm{min}$. The vibration of rotor increases

with the increase of rotating speed. The vibration peak value is $37 \mu \mathrm{m}$, far less than the primary clearance $\pm 0.1 \mathrm{~mm}$.

(2) Electromagnetic levitation force and electromagnetic torque characteristics

Fig. 17 shows the distribution of suspending force and electromagnetic torque which are affected by control current $i_{d}$ and $i_{q}$, separately. $i_{d}$ (suspending force control current) and $i_{q}$ (torque control current)is 28.9A.

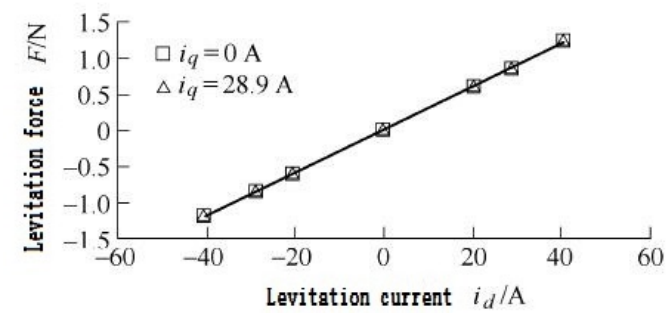

(a)

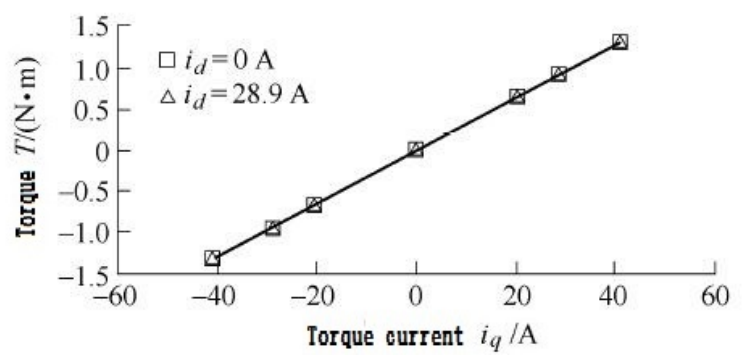

(b)

Fig.17. Distribution of suspending force and electromagnetic torque affected by control current

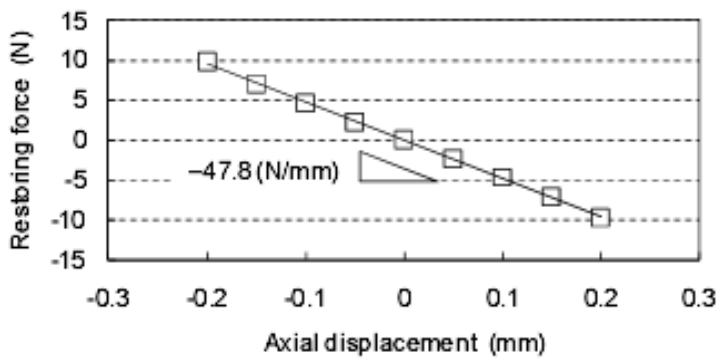

(a)

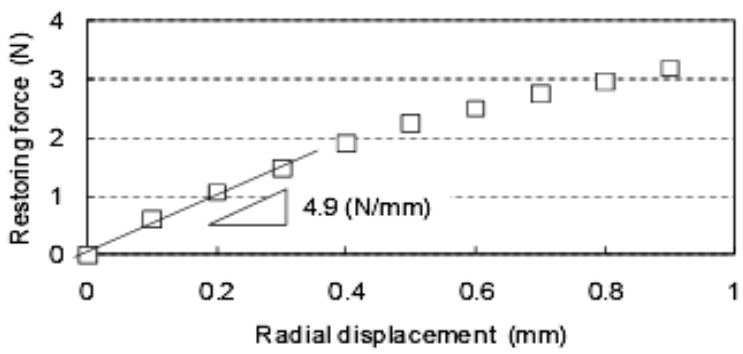

(b)

Fig.18. The changing rule of electromagnetic levitation force of spherical rotor affected by radical displacement

When the torque current $i_{q}$ is 0 and 28.9A, electromagnetic levitation force increases linearly with the increase of control current $i_{d}$, and its changing rate is 
0.030N/A, as shown in Fig.17(a). This illustrates that the change of torque control current doesn't have effects on the performance of electromagnetic levitation force. Similarly, when $i_{d}$ is 0 and $28.9 \mathrm{~A}$, electromagnetic torque increases linearly with the increase of control current $i_{q}$ and its changing rate is $0.032 \mathrm{mNm} / \mathrm{A}$, as shown in Fig. 17(b). This also indicates that the change of force control current doesn't have effects on the performance of electromagnetic torque. So, this experimental control system makes complete decoupling of input and output come true, and even realizes linear control.

Fig.18 shows the changing law of spherical rotor suspending force affected by rotor displacement. Use eddy current displacement sensor to detect rotor displacement and rotor and the rotor's rotating position is detected by two sensors. Voltage source controlled by digital microprocessor is direct voltage of $36 \mathrm{~V}$ that is offered by a 3-phase pulse width modulation inverter, and the inverter's switching frequency is $100 \mathrm{kHz}$. The sampling frequency of suspending control and current control is $300 \mu \mathrm{s}$ and $100 \mu \mathrm{s}$, separately. From the picture, when the spherical rotor deviates from the balance point with a distance of under $0.3 \mathrm{~mm}$, it is usually linear.

\section{Copyright}

Papers submitted to ICISIP must describe original and unpublished researches. They should not have been submitted to other conferences or journals. Any paper which is under review elsewhere will not be considered for publication. Once an eligible paper is accepted, it will be published under a Creative Commons Attribution 3.0

\section{Conclusions}

This paper introduces the fundamental composition and structure features of inductive maglev spherical driving joint. Use the principle of virtual displacement to establish the model of electromagnetic levitation force and electromagnetic torque, based on the distribution law of air gap magnetic field of inductive maglev spherical driving joint. By finite element simulation analysis, verify the distribution of air gap magnetic induction intensity, electromagnetic levitation force and electromagnetic torque of inductive maglev spherical driving joint. Put forward the control circuit of inductive maglev spherical driving joint. Study the characteristics of rotor displacement, electromagnetic levitation force and electromagnetic torque of inductive maglev spherical driving joint.

\section{Acknowledgements}

This work was supported by China Natural Science Foundation for this project (Grant No. 51375427, 51475409), Jiangsu Province Basic Research Program Natural Science Foundation (Grant No.BY2015061-04, BK20141277), Yangzhou city - Yangzhou University of Science and Technology Cooperation Program Funds(No. SXT20140041, YZ2016244), 2016 Six talent peaks project in Jiangsu Province (No. JXQC-030).

\section{References}

(1) MAO Xintao, BAO Gang, YANG Qingjun, “Joint torque control for the pneumatically robotic manipulator with 3 degrees of freedom", Chinese Journal of Mechanical Engineering, Vol.44, No.12, pp.254-260, 2008.

(2) Li Jian, Li Jianfeng, Wu Zhen, "Fault tolerance of redundant manipulators when multi-Joint failed and its optimization", Chinese Journal of Mechanical Engineering, Vol. 38, No.7, pp.111-115, 2002.

(3) ZENG Li, ZHANG Dan, DAI Min, "Maglev spherical reluctance motor with centripetal thrust or pull", China, 200920039032.7 [P]. 2001.

(4) ZENG Li, ZHANG Dan, "Switch reluctance driving joint with magnetic levitation" ,China , 200920039032.7[P]. 2001.

(5) Zeng Li, Wang Jun, Zhang Dan, "Mechanism of magnetic force and electromagnetic torque for spherical reluctance motor with magnetic levitation", China Mechanical Engineering, Vol.2, No.1, pp.208-211, 2011.

(6) Zeng Li, Zhang Fan, Zhang Dan, " Force and torque study of magnetic levitation spherical driving joint with magnetic field segmentation method," Przeglad Elektrotechniczny (Polish), Vol.88, No.7B, pp.235-238, 2012.

(7) Zhang Fan, Zeng Li, Chen Fang, "Study of magnetic levitation spherical joint with decoupling control", Przeglad Elektrotechniczny (Polish), Vol.88, No.7B, pp.239-242, 2012.

(8) Zeng Li, Wang Jun, $\mathrm{Xu}$ Yuanyuan, "Spherical reluctance driving joint with magnetic invitation modeling and inverse system decoupling control", Journal of Mechanical Engineering (in Chinese), Vol.17, No.9, pp.15-21, 2011.

(9) Zeng Li, Zhang Fan, $\mathrm{Xu}$ Yuanyuan, "Three-dimensional dynamical model and controlled characteristics of magnetic levitation spherical reluctance driving Joint", Journal of Mechanical Engineering(in Chinese), Vol.21, No.11, pp.69-75, 2011.

(10) Seiji SHIMURA, Yohji OKADA, Tetsuo OHISHI, Ryou KONDO, "Analysis and control of an induction-type levitated rotating motor". Collected 
